children. J Pediatr Neurol July-Sept 2004:2:125-130). (Respond: Itzhak Brook, MD, MSc, 4431 Albemarle St NW. Washington. DC 20016).

COMMENT. The experience of brain abscess at Children's Hospital, Boston, between 1981 and 2000, is reported by Goodkin et al. 2004 (see Ped Neur Briefs June 2004:18:43-44). Congental heart disease was the most common predisposing factor. In post1981 compared to pre-1981 cases ( $\mathrm{n}=55 \mathrm{cf} 94$ ), the annual rate of abscess was similar, those associated with otitic or sinus infection had decreased in frequency, abscess in infants was more common. acute immunosuppressive disease was a more frequent predisposing factor, treatment with antibiotics alone had become successful in $>20 \%$ cases, but mortality rate had shown no significant change ( $24 \%$ vs $27 \%$ ). Despite improvements in diagnosis using neuroimaging. brain abscess continues to result in high rates of neurologic impairment and death. A greater awareness of presenting symptoms and predisposing factors and earlier diagnosis may be expected to improve outcome in the future. New-onset headache and seizure in a child with congenital heart disease or recent ear or sinus infection, and especially a positive culture for Streptococcus milleri (S intermedius), or fungal disease in an acutely immunosuppressed patient, should alert the physician to the diagnosis of cerebral abscess.

\title{
SEIZURES COMPLICATING BACTERIAL MENINGITIS
}

The clinical data of 116 patients, 1 month to $<5$ years of age, admitted for bacterial meningitis, and grouped according to those with and without seizures during hospitalization, were compared in a study at Buddhist Dalin Tzu Chi General Hospital, Chang Gung Memorial Hospital and other centers in Taiwan. Fifty-five (47\%) had seizures in the acute phase. especially prevalent in the older infants, and 12 of these had one or more afebrile seizures after completing treatment for acute meningitis. Seizures occurring 2 weeks after treatment were associated with hydrocephalus, subdural empyema, or cerebral infarction. Seizure prevalence was high in meningitis caused by $E$ coli, Salmonella species, $S$ agalactiae, Hemophilus species, and $N$ meningitides. At 1-year follow-up, 26 had good outcomes and in 29 the outcome was poor. In those who had seizures in the acute phase, the outcome was worse than those without. Neuroimaging abnormalities correlated with the occurrence of seizures during hospitalization for meningitis. Factors that increased the risk of seizures included disturbed consciousness on admission, abnormal neuroimaging, and low glucose and elevated CSF protein. (Chang C-J, Chang H-W, Chang W-N, et al. Seizures complicating infantile and childhood bacterial meningitis. Pediatr Neurol Sept 2004;31:165171). (Respond: Dr Cheng-Hsien Lu MD, MSc, Department of Neurology, Chang Gung Memorial Hospital, 123, Ta Pei Road, Niao Sung Hsiang, Kaohsiung Hsien, Taiwan).

COMMENT. Seizures are common during hospitalization in the child with bacterial meningitis, and the occurrence of seizures during the acute phase is strongly correlated with late seizures, especially during the first 5 years of follow-up. Treatment of neurologic complications and aggressive antimicrobial therapy are essential for optimal outcome.

Afebrile seizures provoked by minor infections carry a $5.7 \%$ risk of subsequent unprovoked afebrile seizures by 5 year follow-up in a study at National Neuroscience Institute, Singapore (Lee W-L, Ong H-T. Pediatr Neurol 2004;31:157-164). 